\title{
Damage Identification and Performance Assessment of Regular and Irregular Buildings Using Wavelet Transform Energy
}

\author{
Mosbeh R. Kaloop ${ }^{1,2,3}$ and Jong Wan $\mathrm{Hu}^{1,3}$ \\ ${ }^{1}$ Department of Civil and Environmental Engineering, Incheon National University, 12-1 Songdo-dong, Yeonsu-gu, \\ Incheon 406-840, Republic of Korea \\ ${ }^{2}$ Public Works and Civil Engineering Department, Mansoura University, Mansoura 35516, Egypt \\ ${ }^{3}$ Incheon Disaster Prevention Research Center, Incheon National University, 12-1 Songdo-dong, Yeonsu-gu, \\ Incheon 406-840, Republic of Korea
}

Correspondence should be addressed to Jong Wan Hu; jongp24@incheon.ac.kr

Received 9 January 2016; Revised 22 March 2016; Accepted 23 March 2016

Academic Editor: Antônio G. de Lima

Copyright (C) 2016 M. R. Kaloop and J. W. Hu. This is an open access article distributed under the Creative Commons Attribution License, which permits unrestricted use, distribution, and reproduction in any medium, provided the original work is properly cited.

\begin{abstract}
This study investigates a novelty wavelet application for damage detection of regular and irregular building structures under seismic load effects. The energy of wavelet transform and correlation coefficient are used to detect the performance of the damaged building. The simple 3D regular and irregular simulation (finite element model) models of a building are designed to verify the methods' uses and optimum applications. The obtained results reveal that the energy of Discrete Wavelet Transform (DWT) shows significantly higher performance than the energy of Continuous Wavelet Transform (CWT) in detecting the damage of the building, and the performance of irregular buildings appeared suitable for use in the seismically active areas. In addition, it can be concluded that the correlation coefficient can be applied to study the effects of damage and the safety of structures.
\end{abstract}

\section{Introduction}

The structure's damage detection is one of the important parameters for the structural system identifications [1]. Furthermore, the damage is almost detected in the structures in life service [2]. Therefore, it is important to design a monitoring system and develop methods to detect the damage and enhance the safety of structures. The damage occurs due to changes in geometrical or physical properties of structural members [2]. In addition, the damage assessment and identification are divided into four categories, that is, the presence of damage, the location of the damage in the structure, the severity identification of the damage, and the assessment of remaining lifetime of the structure [3]. Thus, the structural health monitoring (SHM) systems are used to measure the performance of structures, to detect and analyze the changes, and to identify the structural condition categorically.
The model identification methods are used to predict the structural movements and damage and assess the performance. Many identifications models are used to detect and localize the damage, and the wavelet transform (WT) is one of those [2,4-6]. WT may be viewed as an extension of traditional Fourier transformation with adjustable window location and size [2, 7]. Furthermore, the WT is considered as an efficient tool for SHM and damage detection [2, 8-10]. Haifeng [3] proposed the advantages of WT in the analyses of structural performance and damage detection. The main advantage of WT is the ability to perform a local analysis of signal measurements $[3,11]$. Thus, it is capable of revealing some hidden aspects of the measurements that other signal analysis techniques fail to detect [11]. Moreover, the WT has emerged as the best tool for the analysis of seismic traces, since it is capable of acquiring high time resolution at higher frequency bands and high-frequency resolution at 
lower frequency bands with an adjustable time-frequency window [3]. This property is particularly cardinal for damage detection applications. Many applications for the structural damage and assessment adopt WT techniques [1-3, 1012]. Most of the applications for damage detection in civil engineering are for regular buildings (experimental study) [12], trusses [9], bridges [6], and beams [8]; therefore, the novelty application for this method is used in this study for the regular and irregular buildings.

The performances of regular and irregular buildings under seismic loads are presented in [13-17]. Also, the response controls of the regular and irregular buildings are presented in [18-20]. The modal analysis of regular building showed that first and third mode shapes had the most dominant modal mass along the longitudinal and transverse directions [17]. Furthermore, the analyses for performance and damage detection of the regular building showed that stochastic damage detection method can be used to detect the damage location and corresponding damage severities when uncertainty or random parameters of the building structure are taken into account [14]. Mazza [16] showed that seismic capacity directions are not necessarily the same at the serviceability and ultimate limit states for the irregular building performance studies. Arangio and Bontempi [21] and Sepúlveda et al. [15] have shown that the output measurements for the structure performance are mostly used to predict the damage of structures. Shao and Tiong [22] expected that more intensity measures could improve the accuracy of the seismic risk estimation. In addition, the nonstructural components which may have an effect on the overall structure integrity for the building members should be studied.

The present study uses a novel application of WT for the regular and irregular buildings' damage detection. The performance of the buildings will be presented in the time domain. The signals coefficient of continuous and discrete methods will be studied. The correlation study of wavelet energy is discussed and the application of correlation is utilized to detect the damage and assess the structures.

\section{Wavelet Theory}

Previously, two Fourier transform- (FT-) based parameters, that is, natural frequencies and mode shapes, have been dominantly used in damage detection and assessment of structural integrity $[3,23]$. WT-based SHM and damage identification is a newly emerging area of studies in the field of SHM. It is known that the response signals of structures usually share the softening trend as the sample signal. In this case, WT provides a new way to analyze the signal, with its merits of "zoom-in" for transient features and "zoom-out" for slow changes [3]. So, by the introduction of WT, both frequency and time information can be captured for signal analyzing. The WT is categorized into Discrete Wavelet Transform (DWT), Wavelet Packet Transform (WPT), Continuous Wavelet Transform (CWT), and Stationary Wavelet Transform (SWT). Herein, the common methods used in the SHM are DWT and CWT. Numerous studies have used different types of the wavelet transform for detecting structural damage, as in $[23,24]$. The fundamental theory of WT is described clearly in $[3,6,23,24]$. The novelty used in this study is the correlation of wavelet energy for the signal to detect and assess the damage and performance of structures and compare between the methods and structure types. However, in this section we will summarize the methodology and application used in this study.

In this study, we will use the continuous and discrete WT methods. As a type of time-frequency domain analysis method, the CWT can transform time domain data into both time and frequency domains. The flexibility from a wide choice of mother wavelets allows the WT to isolate changes in a signal that may be difficult to detect using other transform methods such as Fourier transforms. The CWT of a set of time domain signals $x(t)$ with respect to the mother wavelet function $\varphi(t)$ is shown in $[6,24]$. Consider

$$
C(i, j)=\frac{1}{\sqrt{i}} \int_{-\infty}^{\infty} x(t) \varphi^{*}\left(\frac{t-j}{i}\right) d t,
$$

where $C$ indicates wavelet coefficients of CWT; $i$ and $j$ are the scale and translation parameters; and $\varphi^{*}(f)$ represents the complex conjugate of the mother wavelet $\varphi(f)$. When using the FT, one usually develops the spectrogram. The WT has scaling factors. The analog to spectrogram is the scalogram, defined as

$$
|C(i, j)|^{2} .
$$

The scalogram is a measure of the energy distribution over time shift $j$ and scaling factor $i$ of the signal. It holds that the total energy $E_{T}$ of a signal $x(t)$ is

$$
E_{T}=\int_{t} x(t)=\sum_{j} \sum_{i}|C(i, j)|^{2}
$$

where $m$ and $n$ are the order of scale and translation parameters.

The CWT coefficients of signal damaged and undamaged locations of the structure, transformed by the mother wavelet, were compared in contour plots. When the damage was introduced in the structure, peak values of the wavelet coefficients can be observed to shift from higher frequency levels into lower frequency levels in frequency domain. In addition, the wavelet coefficients are observed to be dispersed in time domain. Also, the correlation between the energies of wavelet coefficient for the undamaged and damaged cases is calculated to assess and detect the damage.

On the other hand, the DWT is a linear transformation that operates on a data vector. It is a tool that separates data into different frequency components that are the approximations and the details. The produced low-pass portion of the signal is called the "approximations." They are the highscale and low-frequency components of the original signal. However, the "details" are the high-pass portion which is the low-scale and high-frequency component of the original signal. The decomposition of the original signal using the wavelet analysis is expressed as

$$
x_{k}(t)=A_{k+1}(t)+D_{k+1}(t),
$$


where $A(t)$ is the approximations component, $D(t)$ is the details component, and $k$ is the level number representing the particular range of frequency content of the signal.

The original signal can be reconstructed from the details only without any significant loss of information $[25,26]$; and the detail components of the original signal can be expressed by the linear combination of the mother wavelet as follows:

$$
D_{k}(t)=\sum_{-\infty}^{\infty} C_{k, j} \varphi_{k, j}
$$

where $j$ represents an index of time scale, $C_{k, j}$ are the corresponding wavelet coefficients, and $\varphi_{k, j}$ are the mother wavelet.

Based on the previous calculations, the total energy of a discrete signal is the sum of the squares of its values and, therefore, the total energy of the original signals or decomposition is as follows:

$$
E_{T}=\sum_{k} \sum_{t}\left|D_{k}(t)\right|^{2} .
$$

The DWT can be used to pinpoint the energy of a signal within a particular frequency band. The DWT also provides time resolution; each subband is a time domain signal, allowing for changes in frequency content over time to be detected. This property is important for the real-time monitoring of structures to detect damage as it happens. In addition, the DWT has advantages when the signals being processed are not comprised of pure sinusoids. The signals used in SHM have most of their energy at the natural frequencies of the structure, and decomposition should be done such that the energies at these natural frequencies are confined to specific subbands. Then, if damage to a structure causes a natural frequency of the structure to shift, that damage will be seen clearly as a change in the energy in the corresponding subband. As the same in CWT, the correlation between the energies of discrete wavelet is applied to assess and detect the damage.

\section{Simulation and Seismic Design}

3.1. Structural Model. A simulated 3D 3-story momentresisting steel frame building structure with a regular plan on each floor and a total height of $930 \mathrm{~mm}$ as shown in Figures 1(a) and 1(b) is designed using Abaqus finite element program. A model was generated according to the properties of previous studies for the shaking table experiments $[19,20]$. Diameter of circle section area of steel columns is $5 \mathrm{~mm}$ and story height is $310 \mathrm{~mm}$ in every story. All degrees of freedom (DOFs) are restricted in base level. Each floor consists of a rigid steel square plate $(300 \times 300 \mathrm{~mm})$ with $3420 \mathrm{gr}$ weight with three degrees of freedom, translation in $x$ - and $y$-directions, and rotation around a vertical line passing through centers of mass in each floor. Therefore, total number of DOFs after application of the boundary conditions, rigid diaphragm, and also Guyan reduction of vertical DOFs and rotational DOFs around $x$ - and $y$-axes is equal to 9 ; however, it was equal to 42 prior to boundary
TABLE 1: The properties of used materials in simulation of structural members.

\begin{tabular}{lcc}
\hline Item & Value & Unit \\
\hline Young's modulus & $2.07 \times 10^{6}$ & $\mathrm{MPa}$ \\
Poisson's ratio & 0.30 & - \\
Density & 7.60 & $\mathrm{~g} / \mathrm{cm}^{3}$ \\
Ultimate strength & 400 & $\mathrm{MPa}$ \\
\hline
\end{tabular}

conditions. Each irregular floor is designed by attaching a mass ( $m=420 \mathrm{gr})$ in specific part with steel plate weight $3000 \mathrm{gr}$; this simulation is designed to compare the effect of same mass in each floor in between regular and irregular buildings systems. Thus, the total weight of each floor in the two cases will be equal to 3420 gr (as shown in Figures 1(b) and $1(\mathrm{c})$ ). The plan eccentricity of irregular case is provided by 2.5 and $6.14 \%$ in each floor in $x$ - and $y$-directions, respectively (Figure 1(c)). Table 1 lists the properties of materials used in members of structural model.

3.2. Seismic Load and Damage Level. The regular and irregular buildings are proposed in Figure 1. The three points F1, F2, and F3 are monitored and the acceleration response for the three points is measured. The undamaged case is assumed with all elements of columns being $5 \mathrm{~mm}$. Other than the intact structure, two damage cases are studied for both buildings: (D1) 40\% stiffness loss of the column F2-F3 element and (D2) $80 \%$ stiffness loss of the same element. To assess the seismic performance of buildings cases, a dynamic loading is applied to the structure. A fault actual ground motion measured by strong motion instruments during the Los Angeles (USA) earthquake is selected. The employed shaking provides capability to apply excitation load in the $x$-direction. Figure 2 presents the input signal, frequency domain power spectral density, and response spectra of excitation signal with 5\% damping and 1.0 scale factor (SF). In addition, peak ground motion considered is presented in Figure 2(a); and fundamental periods of vibration for undamaged regular and irregular models, which are estimated by using the computational command provided in the Abaqus program, are 0.0910 and $0.0907 \mathrm{Sec}$, respectively. As it is clear from Figure 2(c), the dominant frequency of seismic load is $1.57 \mathrm{~Hz}$; in addition, it can be seen that the provided effective frequency range is between 0.6 and $15.87 \mathrm{~Hz}$.

\section{Results and Discussions}

The response assessment and damage detection of building consist of frequency changes and mode shapes, as presented previously. The percentages of relative changes of frequencies to the regular mode for fifth mode of finite element model (FEM) of the building are shown in Figure 3(a). From this figure, it can be seen that the irregular building is more controlled than the regular building in the undamaged case. Furthermore, it can be seen that the frequencies changes of damage cases D1 and D2 are decreased by $6 \%$ on the first two modes and increased from 12.9 to $14.2 \%$ on the fourth mode. In addition, it can be seen that the maximum frequency 


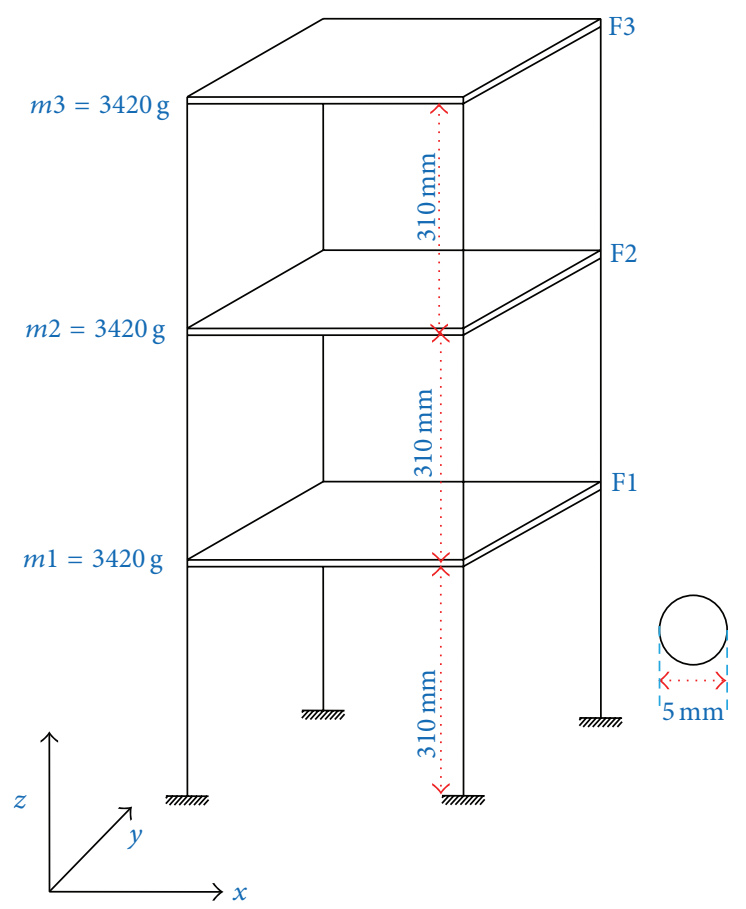

(a) 3D regular steel frame

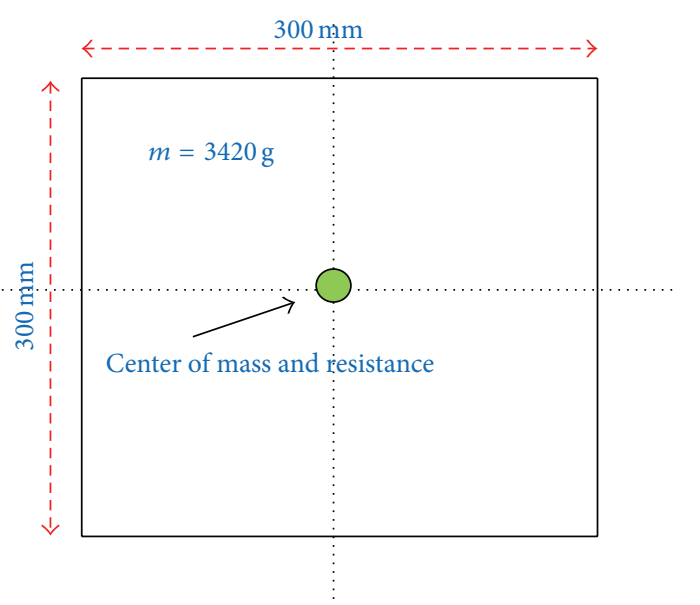

(b) Regular plan for each floor

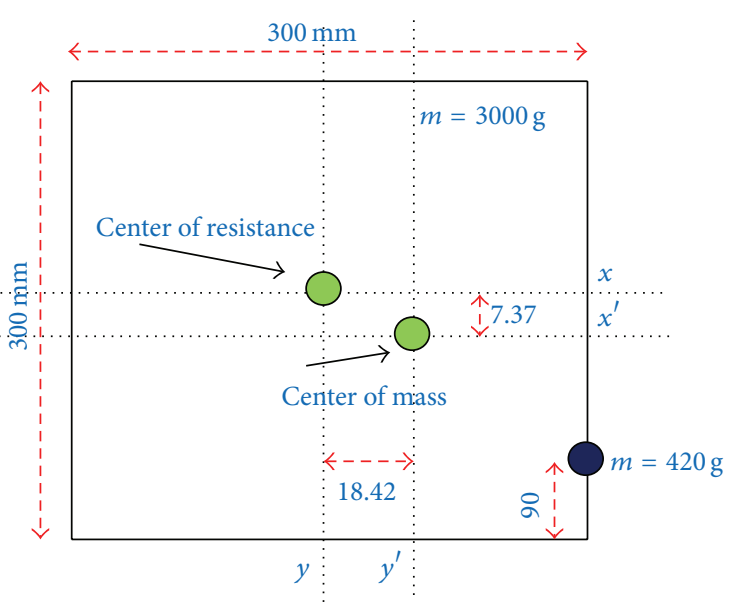

(c) Irregular plan for each floor

FIGURE 1: Simulated regular and irregular 3D three-story resisting steel frame building structure (unscale dimensions).

change occurred with irregular cases. This implies that the irregular building is more uncontrolled with damage cases; this can be used for emergence of the human used building in the earthquake that occurred. Herein, we recommended that the best model design is irregular building in the seismic areas, more control in the undamaged case, and high emergency in damaged case. The high power frequency and high power spectrum density (PSD) of measured acceleration of point F3 are shown in Figure 3(b). From this figure, it can be seen that the measured frequency changes are increased clearly for the regular and irregular cases. Also, it can detect the damage of structures based on the values of power spectrum of signals measurements. In addition, it can be observed that the PSD of regular case is decreased with damage effect while the opposite occurred with irregular case. Therefore, it can be concluded that the measurement response is found to be a good tool to detect the performance and damage of structures. From this result, the significance of structural health monitoring advantages can be understood.

4.1. Continuous Wavelet Analysis. This section discusses results of WT for the buildings, regular and irregular, in vibration and damage, D1 and D2, to study the effectiveness of WT energy methods and predict the damage. The Daubechies wavelet (DB10) was used in both Continuous Wavelet Transform and Discrete Wavelet Transform. Figure 4 


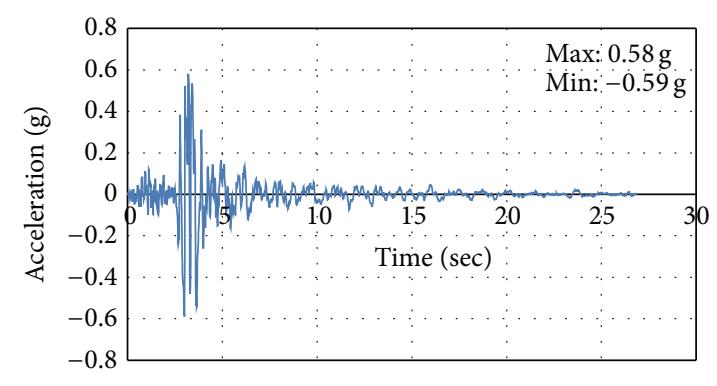

(a)

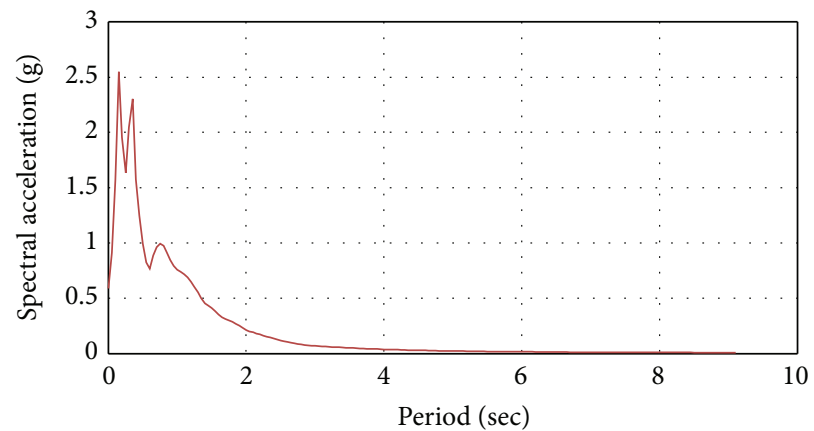

(b)

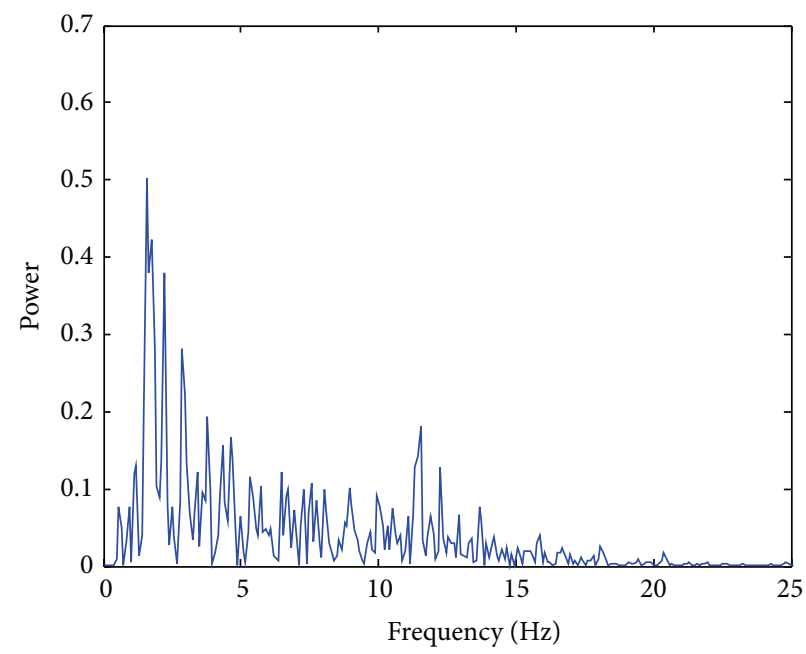

(c)

FIgURE 2: (a) Seismic excitation signal, (b) spectral accelerations, and (c) power spectral density of selected ground motion.

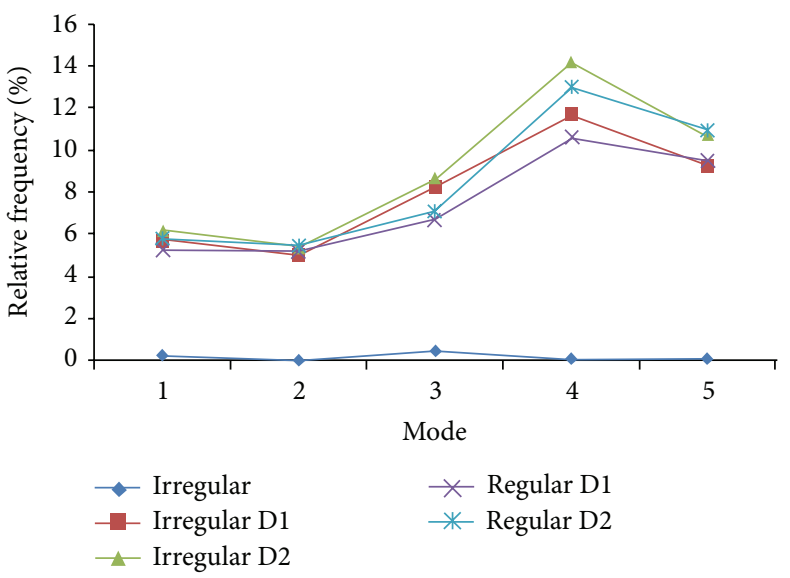

(a) FEM frequency changes

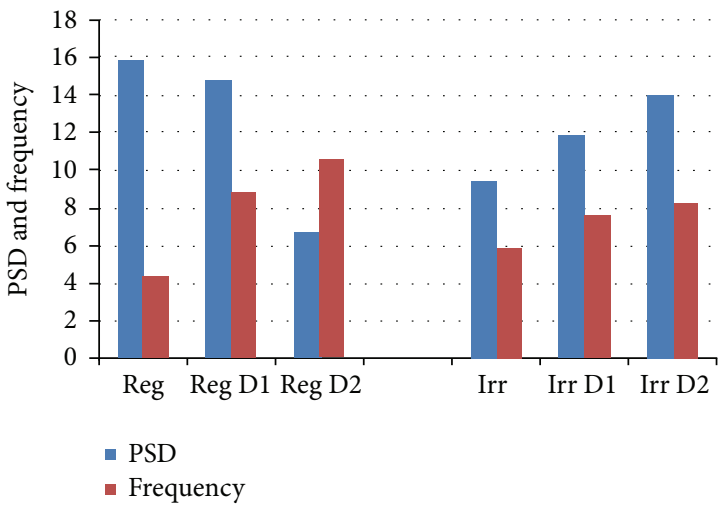

(b) Monitoring PSD and frequency

FIgURE 3: The relative frequency change for the FEM model and PSD for the measurement acceleration.

illustrates the continuous wavelet coefficients (CWC) of F3 for the undamaged and damaged cases of the regular and irregular buildings. From Figure 4, it can be seen that the distribution of CWC on the time for the regular building is greater than the distribution of CWC for the irregular building. In addition, it can be shown that the high CWC is observed at 4.5 and $5 \mathrm{sec}$ for the regular and irregular buildings, respectively. Moreover, it can be seen that the CWC for the irregular building is lower than regular one after the high amplitude of acceleration release. The comparison 

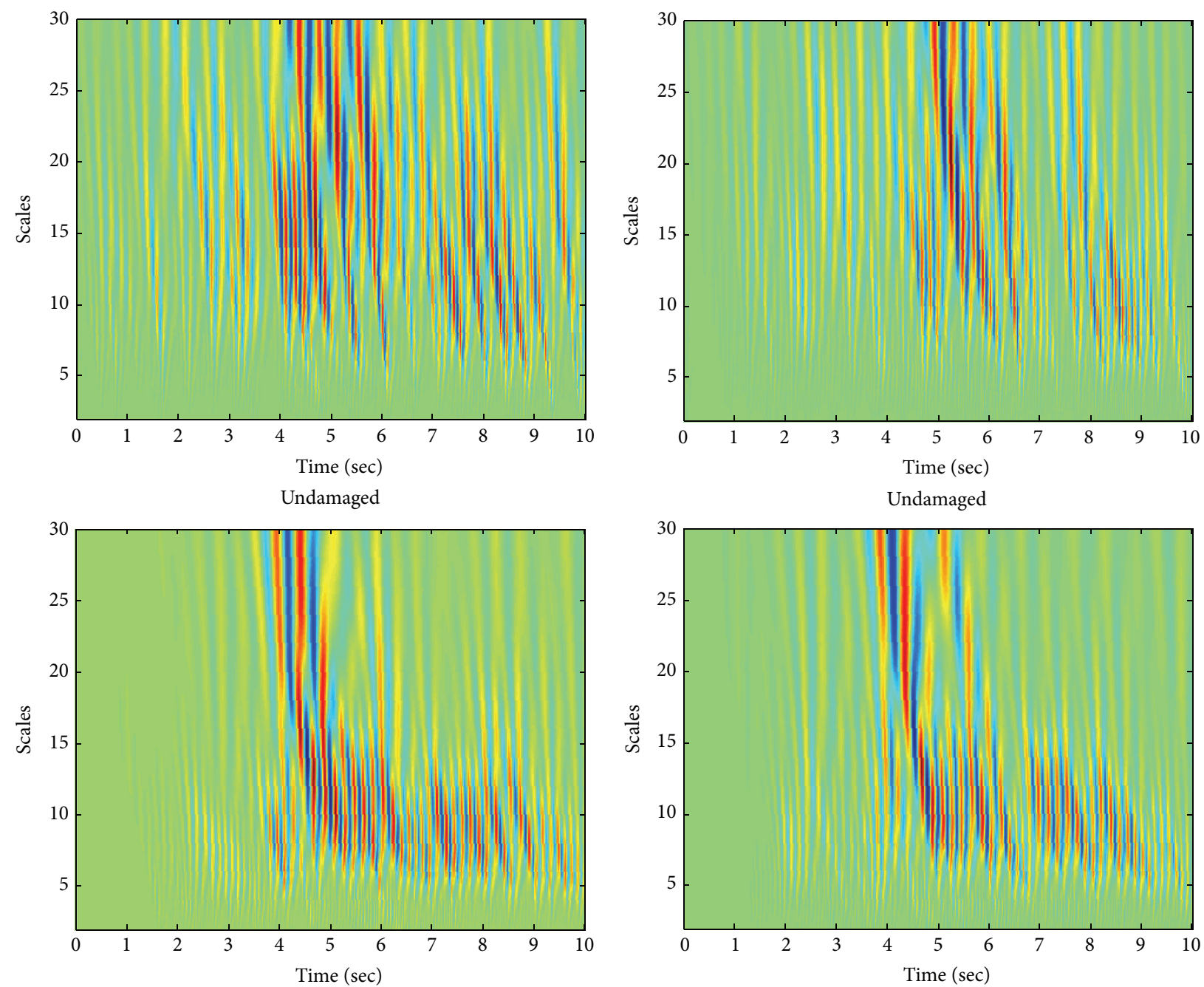

D1
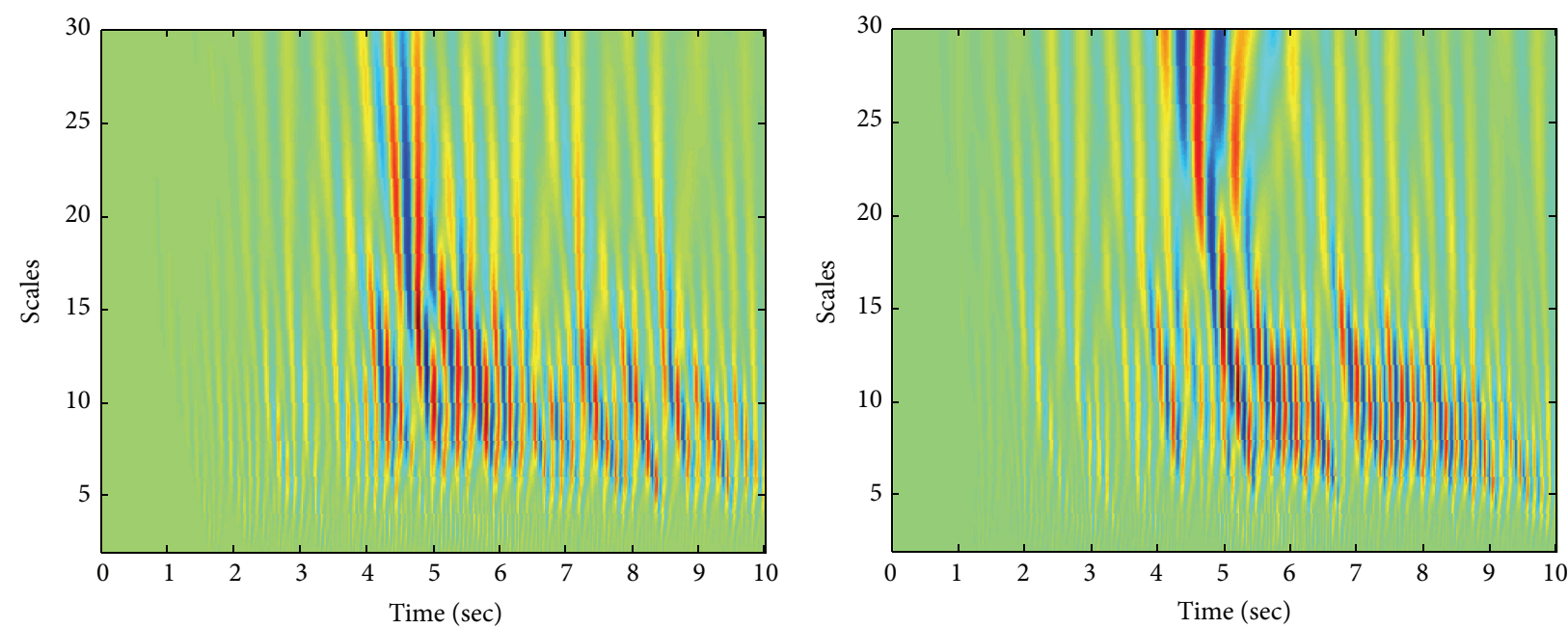

D2

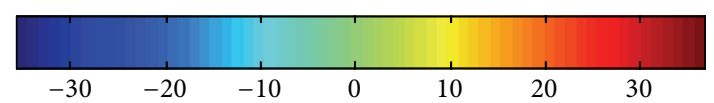

(a)

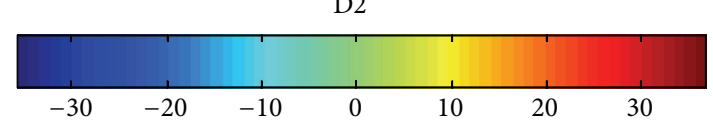

(b)

FIGURE 4: Continuous wavelet coefficients of F3 behavior during shaking effect for regular (a) and irregular (b) buildings. 


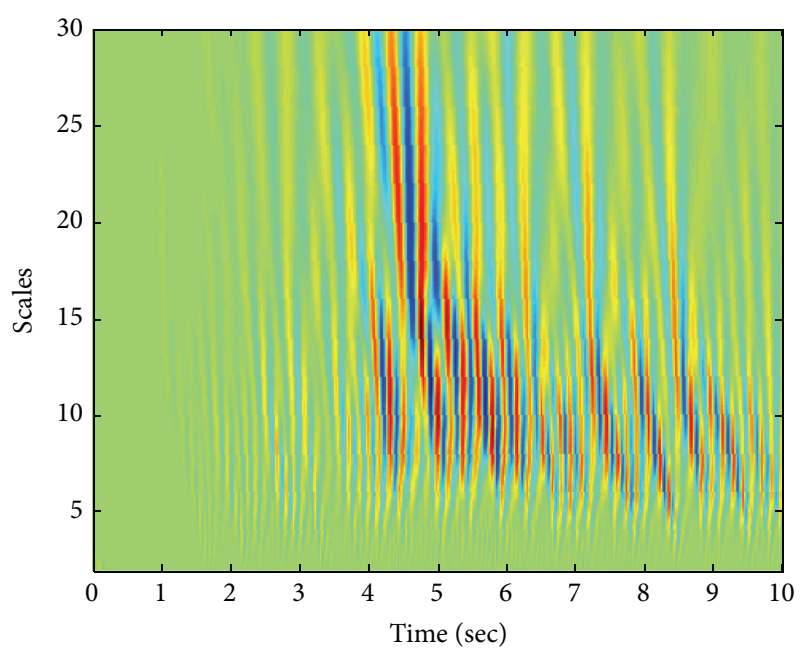

F2

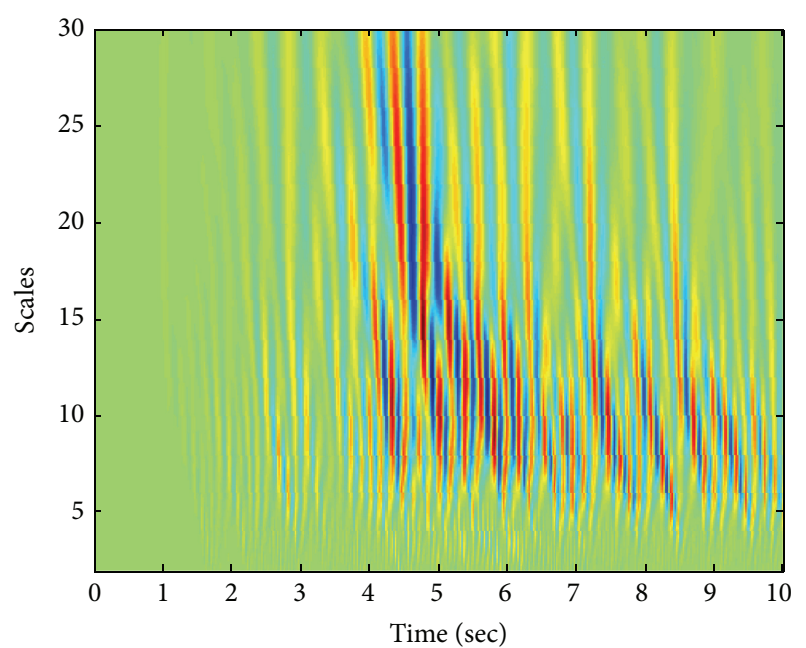

F1

FIGURE 5: Regular damage D2 performance floors.

of CWC for the two buildings has shown that, also, the performance of regular building during and after shaking has taken longer time than irregular building. Therefore, it means that the irregular building is better than the regular one in the performance during shaking effects. From the comparison between damage D1 and undamaged case for the two building cases, it can be seen that the CWC is higher with irregular damage case and the high coefficients are observed at $3.7 \mathrm{sec}$. In addition, it can be observed that the time performance for the irregular building in the damage case is greater than regular case. The performance of building due to damage is shown on the CWC density. Furthermore, it can be shown that the performance of two buildings is approximately the same after the maximum amplitude of shaking effects. Similarly, from the comparison between the D2 and D1 cases, it can be seen that the lower CWC (10) is observed in the case of D2. In addition, it can be shown that the coefficients amplitude is increased as the damage increased.

From these results, it can be concluded that the irregular building has more stability and lower performance than regular case in undamaged case. The two cases of building are approximately the same in performance in damage case, while the irregular building is shown to be the more emergent one. It means that the recommended case of building in the seismic zones is irregular one. The CWC has shown high sensitivity for the shaking effects and can be used to detect the damage. In addition, the lower coefficients amplitude should be considered to define the damage level.

Figure 5 presents the performance of F1 and F2 for the regular building in the case of damage D2. From Figures 5 and 4 (regular floor F3), it can be seen that the performances of three floors are approximately the same, but it can be observed that the high CWC occurred at F1 and after that the floors F2 and then F3. It implies that the CWC can be used to detect the damage location.

Figure 6 illustrates the total energy of CWT of regular and irregular buildings. From Figure 6, it can be seen that the energies of three floors in the two cases, regular and irregular, are highly correlated (0.99) in the undamaged case. In addition, it can be seen that the high energies of three floors are increased with damage cases D1 and D2. Moreover, the high energy scale is decreased when damage occurs. Also, it can be observed that the correlation between energies for F2 and F3 is 0.99 and 0.96 in damage D2 for the regular and irregular buildings, respectively, while the correlations between F2, F3, and F1 are 0.99 and 0.99 for the regular building and 0.98 and 0.93 for the irregular building. Also, it can be seen that the correlation between undamaged and damaged cases is low (0.60 and 0.63 for the regular and irregular buildings, resp.). It infers that the correlation coefficient of energy can be used to detect the damage and location. Furthermore, the maximum energy has occurred at F2 and F3 (damage location). It means that the high energy refers to the damage occurrence.

From the comparison between two cases of building, it can be seen that the performances of total energies of buildings are approximately the same with the change of maximum energy (at scales 13 and 24 for regular and irregular buildings, resp.). In addition, it can be seen that the performance of energy contents of irregular case reverts back to the performance of undamaged case more than the regular case. Moreover, the correlation between F1 and F2 and between F1 and F3 for the irregular case is shown to be lower than the regular case. Furthermore, it can be observed that the maximum energy occurred with D1 in the regular case while it occurred with D2 in the irregular case. This means that the irregular buildings are more stable and emergent with seismic shaking effects.

4.2. Discrete Wavelet Analysis. The approximation and detail decomposition of DWT of the acceleration signal of regular F3 from level 1 to level 6 are presented from top to bottom in Figure 7. The Matlab function "wmaxlev" is used to calculate the number of decomposition levels. The levels of regular and irregular buildings are found in Figures 6 and 7, 


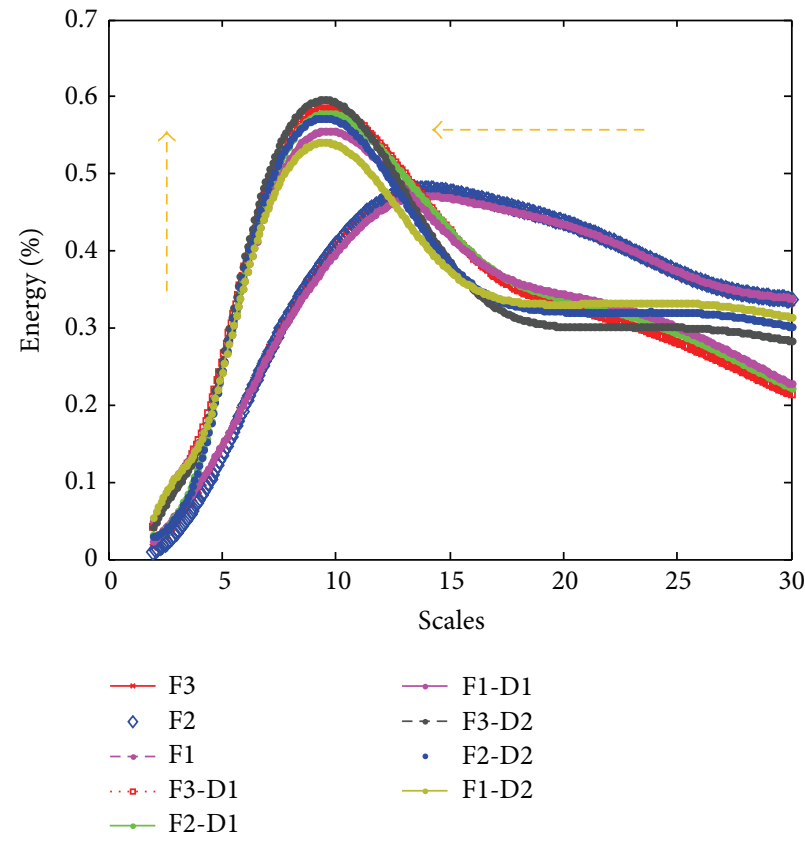

(a)

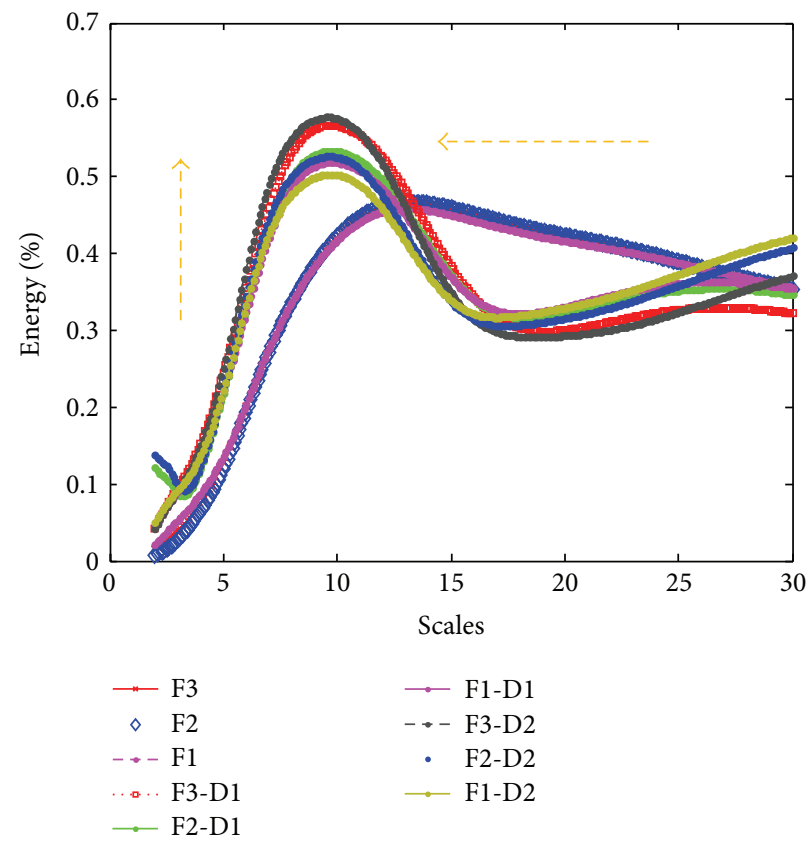

(b)

FIgURE 6: Total wavelet energy of (a) regular and (b) irregular buildings.

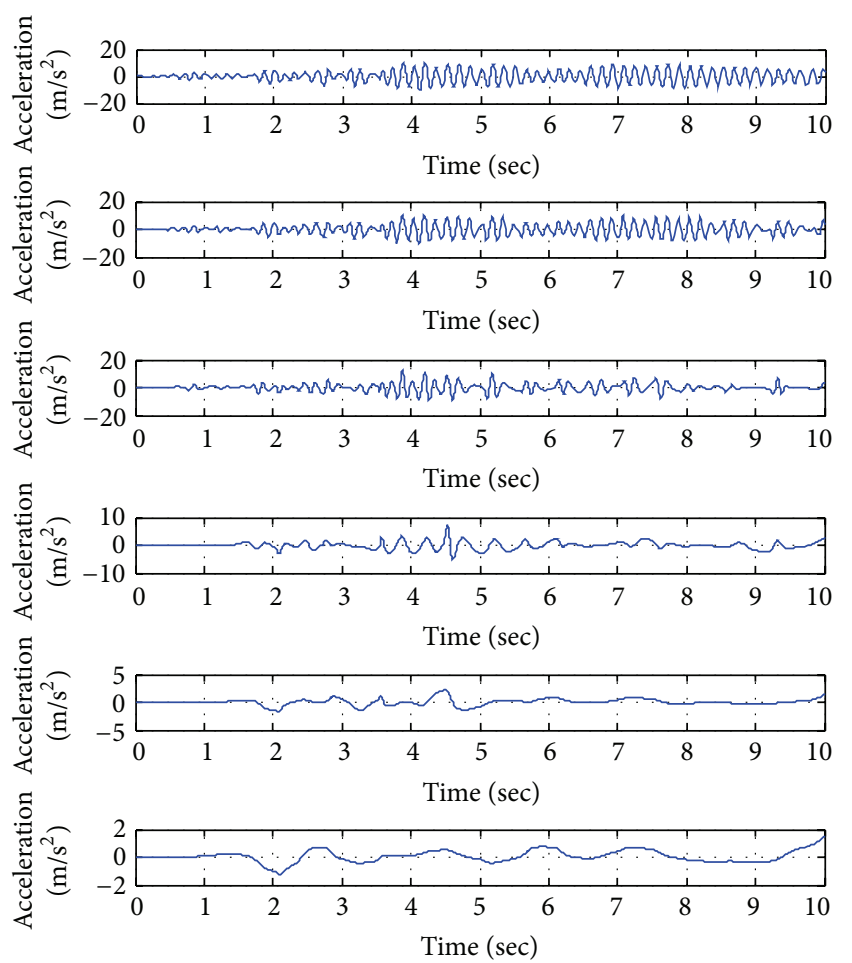

(a)

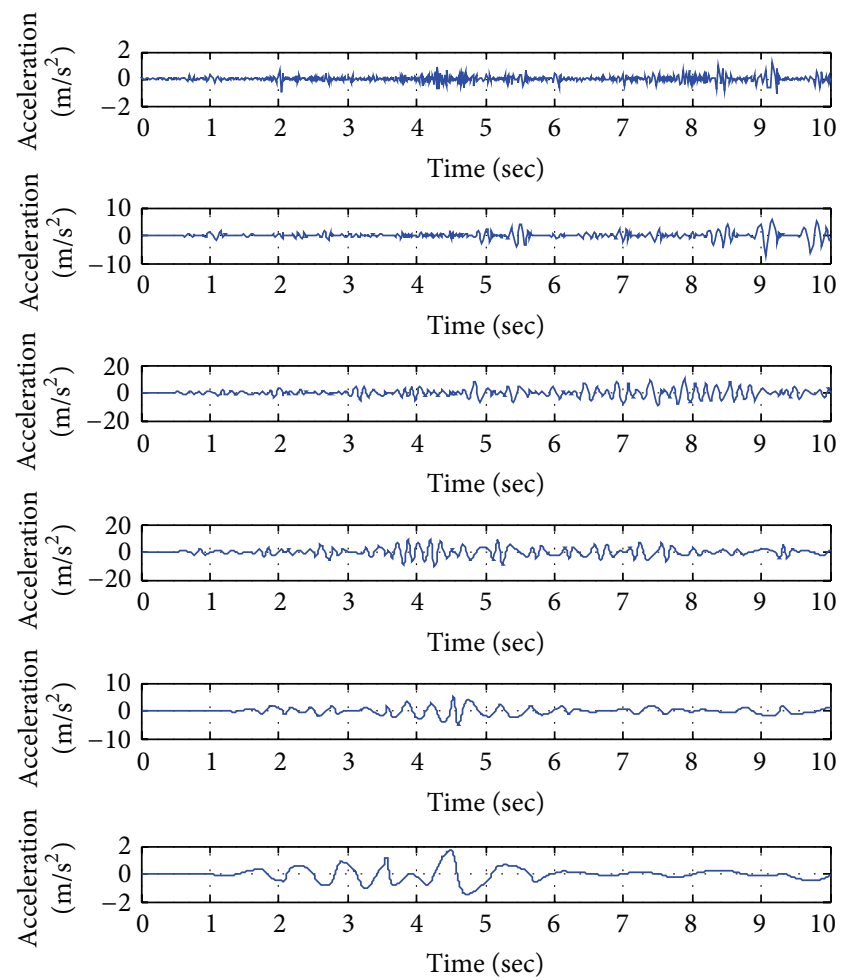

(b)

FIGURE 7: The regular (a) approximate and (b) detail decomposition DWT of F3 building. 


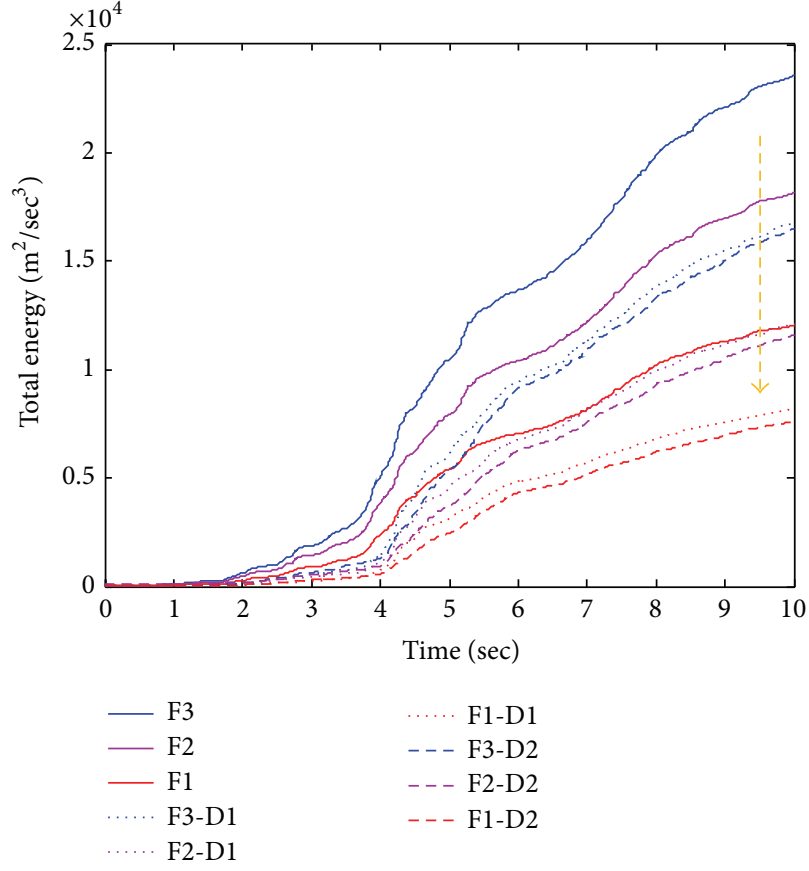

(a)

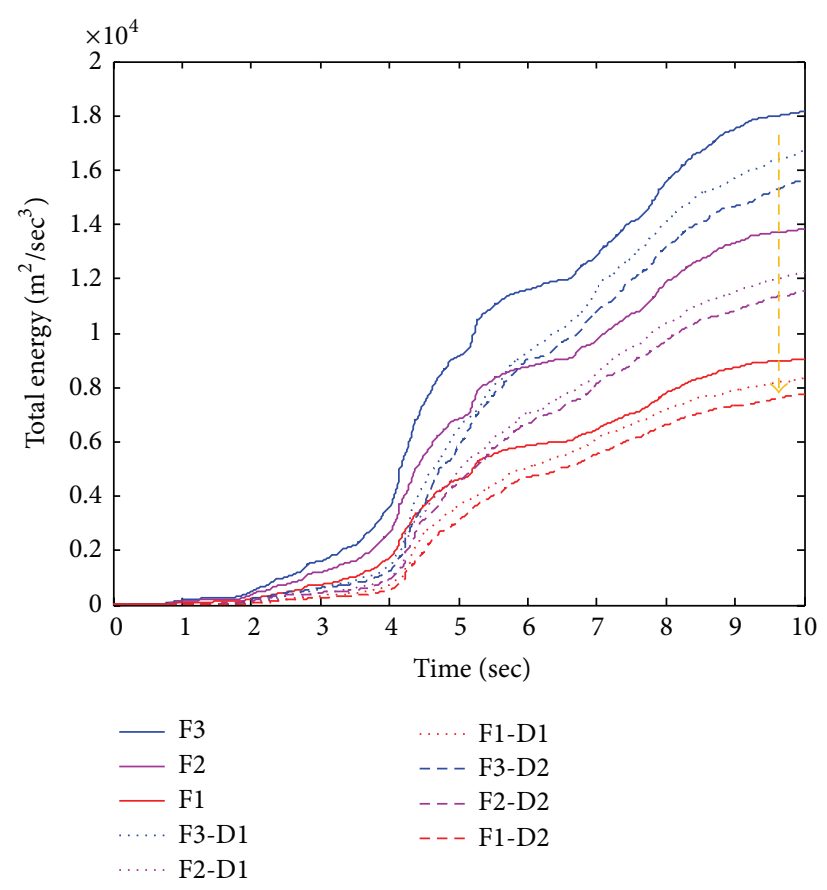

(b)

FIGURE 8: Total energy of (a) regular and (b) irregular buildings during damage cases D1 and D2.

respectively. Therefore, the 7th level is used to calculate the wavelet energy.

The total energy of building based on DWT is presented in Figure 8. Figure 8 illustrates that the total energy of F3 is higher than F2 and F1 in the two cases of buildings. In addition, it can be shown that the maximum total energies for the regular and irregular buildings are $2.3 e 4$ and $1.8 e 4 \mathrm{~m}^{2} / \mathrm{sec}^{3}$, respectively. Furthermore, it can be seen that the total energy of regular damage $\mathrm{D} 1$ is decreased by $27.4,33.3$, and $32.3 \%$ on F3, F2, and F1, respectively. Also, damage D2 is decreased on F3, F2, and F1 by $30.4,38.8$, and 37.0\%, respectively. Moreover, the total energy of irregular damage cases D1 and D2 is affected by $5.6,14.3$, and $7.8 \%$ and $11.1,17.8$, and $14.2 \%$ for the different roofs (F3, F2, and F1), respectively. From this figure, it can be concluded that the two building types are affected by different damage, but it can be seen that the irregular building is more emergent and safe in the seismic areas. In addition, it can be seen that the correlations between the total energies of levels for the undamaged and damaged cases D1 and $\mathrm{D} 2$ for the $\mathrm{F} 3$ of the regular building are 0.987 and 0.983 , respectively. In the same case for the irregular one, it is shown that the correlations are 0.956 and 0.931 . The correlation for F2 is shown to be 0.987 and 0.939 for the regular and irregular buildings, respectively, at damage D1. As the same damage case, it can be seen that the correlations for regular and irregular buildings are 0.982 and 0.935 , respectively, for the F1 floor. From the correlation value, it can be concluded that the correlation values can be used to detect the performance of buildings and classify the types of building while it can be seen that the regular one has higher correlation than the irregular one. So, based on the degree of the irregularity, the correlation should be decreased.

The energy of each level of DWT is illustrated in Figure 9. From this figure, it can be seen that the dominant levels for the regular building are 4 by $49.5 \%$ then level 3 by $32 \%$. Moreover, the levels of the irregular building are shown to be dominant at levels 3 and 4 by 40.3 and $46 \%$, respectively. The performance damage of two buildings is shown to be approximately similar to the two damage cases; the damage cases are dominant at level 3. In addition, it can be shown that the maximum energy of the irregular building is smaller than the regular one by $8 \%$. In addition, the correlation between the distributions of undamaged and damaged D1 cases has shown that the correlations of F3 floor for regular and irregular buildings are 0.572 and 0.649 , respectively. It means that the distribution of the irregular building and energy is more suitable than regular one in the cases of damage. Moreover, with damage, the roof response correlation trend decreases dramatically for all frequency level contents except for the lowest level which suffers less decrease; however the correlation coefficient of the highest frequency level (levels one and two) increases with the increasing damage.

The comparison of CWT and DWT in the two cases shows that the total energy of continuous and distribution of discrete levels have observed the damage and can be used to detect the damage. In addition, it can be seen that the correlation coefficient values for the discrete are lower than continuous one. Furthermore, the total energy of discrete method is clearer than the continuous one. Therefore, it can be concluded that the discrete wavelet energy is more suitable 


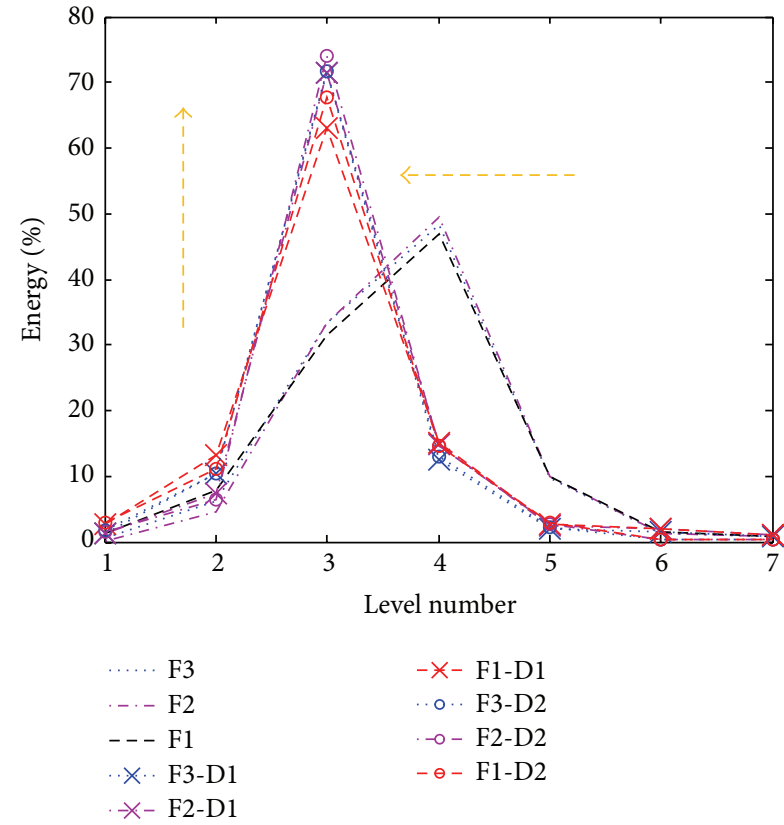

(a)

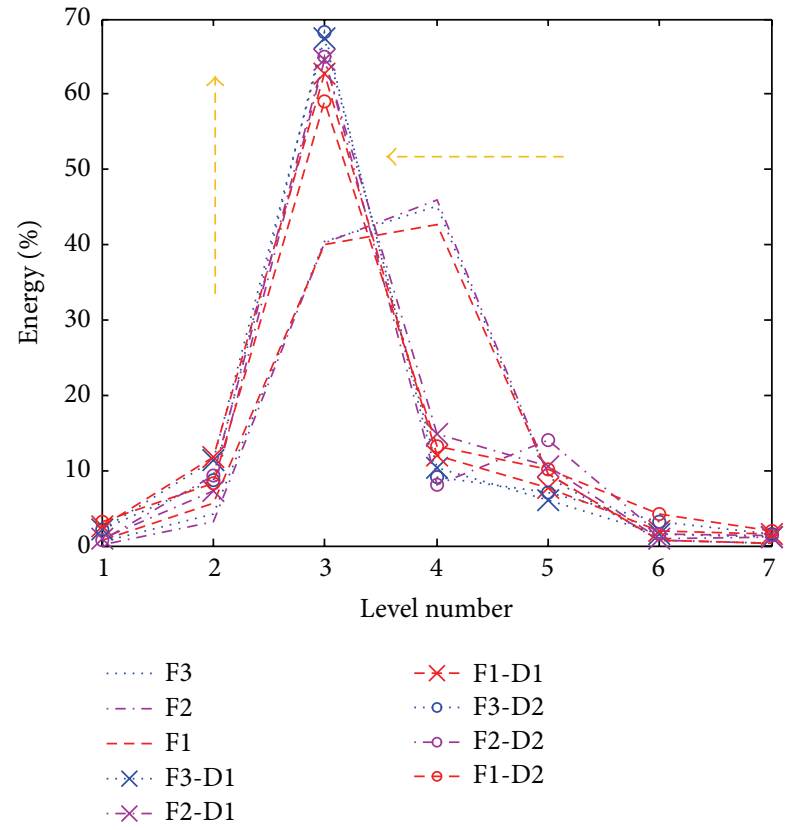

(b)

FIGURE 9: Energy distributions at each decomposition level of (a) regular and (b) irregular buildings under different damage cases.

to detect the damage and study the effect of damage. Also, it can be noted that the correlation coefficient can be used to study the effects of damage and study the safety of structures.

\section{Conclusions}

The wavelet transform is proposed as a damage detection and performance analysis method to study the regular and irregular buildings behavior. To investigate the performance of the proposed method and buildings types, two different $3 \mathrm{D}$ modular steel structures are designed using FEM. The new damage detection and performance analysis methods are proposed using wavelet energy of wavelet contents. Two wavelet energy contents of CWT and DWT are used in this study. The correlation coefficient used in detecting structural (regular and irregular buildings) damage and performance was applied to find optimum methods of the wavelet to classify the damage of structures. The energy of DWT shows significantly higher performance than the energy of CWT in detecting damage of building, which has little and high effect on the model properties of buildings types. The performance of regular and irregular buildings during seismic loads effects and damage detection of two types of buildings show that the irregular buildings are suitable to use in seismically active areas. In addition, it can be noted that the correlation coefficient can be used to study the effects of damage and study the safety of structures based on the energy of wavelet transform. Finally, an experimental work and field observation are needed to complement the real analytical studies.

\section{Competing Interests}

The authors declare that they have no competing interests.

\section{Acknowledgments}

This research was supported by Basic Science Research Program through the National Research Foundation of Korea (NRF) funded by the Ministry of Science, ICT, and Future Planning (Grant no. 2013R1A2A2A01068174).

\section{References}

[1] G. F. Sirca Jr. and H. Adeli, "System identification in structural engineering," Scientia Iranica, vol. 19, no. 6, pp. 1355-1364, 2012.

[2] Z. Hou, M. Noori, and R. St Amand, "Wavelet-based approach for structural damage detection," Journal of Engineering Mechanics, vol. 126, no. 7, pp. 677-683, 2000.

[3] W. Haifeng, A wavelet based damage identification for ship building gantry cranes [M.S. thesis], California Polytechnic State University, San Luis Obispo, Calif, USA, 2015.

[4] H. Kim and H. Melhem, "Damage detection of structures by wavelet analysis," Engineering Structures, vol. 26, no. 3, pp. 347362, 2004.

[5] M. M. R. Taha, A. Noureldin, J. L. Lucero, and T. J. Baca, "Wavelet transform for structural health monitoring: a compendium of uses and features," Structural Health Monitoring, vol. 5, no. 3, pp. 267-295, 2006.

[6] M. R. Kaloop and H. Li, "Sensitivity and analysis GPS signals based bridge damage using GPS observations and wavelet transform," Measurement, vol. 44, no. 5, pp. 927-937, 2011. 
[7] Z. Sun and C. C. Chang, "Structural damage assessment based on wavelet packet transform," Journal of Structural Engineering, vol. 128, no. 10, pp. 1354-1361, 2002.

[8] W.-X. Ren and Z.-S. Sun, "Structural damage identification by using wavelet entropy," Engineering Structures, vol. 30, no. 10, pp. 2840-2849, 2008.

[9] F. Zhu, Z. Deng, and J. Zhang, "An integrated approach for structural damage identification using wavelet neuro-fuzzy model," Expert Systems with Applications, vol. 40, no. 18, pp. 7415-7427, 2013.

[10] M. Makki Alamdari, J. Li, and B. Samali, "Damage identification using 2-D discrete wavelet transform on extended operational mode shapes," Archives of Civil and Mechanical Engineering, vol. 15, no. 3, pp. 698-710, 2015.

[11] S. S. Law, X. Y. Li, X. Q. Zhu, and S. L. Chan, "Structural damage detection from wavelet packet sensitivity," Engineering Structures, vol. 27, no. 9, pp. 1339-1348, 2005.

[12] Y. Yang and S. Nagarajaiah, "Blind identification of damage in time-varying systems using independent component analysis with wavelet transform," Mechanical Systems and Signal Processing, vol. 47, no. 1-2, pp. 3-20, 2014.

[13] R. K. Goel, "Seismic response control of irregular structures using nonlinear dampers," in Proceedings of the 13th World Conference on Earthquake Engineering, Paper no. 3212, Vancouver, Canada, August 2004.

[14] Y. L. Xu, J. Zhang, J. Li, and X. M. Wang, "Stochastic damage detection method for building structures with parametric uncertainties," Journal of Sound and Vibration, vol. 330, no. 20, pp. 4725-4737, 2011.

[15] C. Sepúlveda, J. C. De la Llera, and A. Jacobsen, "An empirical model for preliminary seismic response estimation of freeplan nominally symmetric buildings using ANFIS," Engineering Structures, vol. 37, pp. 36-49, 2012.

[16] F. Mazza, "Modelling and nonlinear static analysis of reinforced concrete framed buildings irregular in plan," Engineering Structures, vol. 80, pp. 98-108, 2014.

[17] J. Seo, J. Hu, and B. Davaajamts, "Seismic performance evaluation of multistory reinforced concrete moment resisting frame structure with shear walls," Sustainability, vol. 7, no. 10, pp. 14287-14308, 2015.

[18] O. Yoshida and S. J. Dyke, "Response control of full-scale irregular buildings using magnetorheological dampers," Journal of Structural Engineering, vol. 131, no. 5, pp. 734-742, 2005.

[19] Y. Bigdeli and D. Kim, "Damping effects of the passive control devices on structural vibration control: TMD, TLC and TLCD for varying total masses," KSCE Journal of Civil Engineering, vol. 20, no. 1, pp. 301-308, 2015.

[20] Y. Bigdeli and D. Kim, "Active control of 3-D irregular building by using energy based neuro-controller active control of 3D irregular building by using energy based neuro-controller," Advances in Structural Engineering, vol. 17, no. 6, 2015.

[21] S. Arangio and F. Bontempi, "Bayesian neural networks for damage identification of a cable-stayed bridge," in Bridge Maintenance, Safety, Management, Resilience and SustainabilityProceedings of the Sixth International Conference on Bridge Maintenance, Safety and Management, pp. 2260-2266, 2012.

[22] Z. Shao and R. L. K. Tiong, "Estimating potential economic loss from damage to RC buildings in Singapore due to far-field seismic risk," Journal of Earthquake Engineering, vol. 19, no. 5, pp. 770-783, 2015.
[23] S. Lee, Hybrid damage identification based on wavelet transform and finite element model updating, [Doctor of Philosophy, Civil Engineering thesis], University of Akron, Akron, Ohio, USA, 2012.

[24] R. Büssow, "An algorithm for the continuous Morlet wavelet transform," Mechanical Systems and Signal Processing, vol. 21, no. 8, pp. 2970-2979, 2007.

[25] B. Cazelles, M. Chavez, D. Berteaux et al., "Wavelet analysis of ecological time series," Oecologia, vol. 156, no. 2, pp. 287-304, 2008.

[26] Z. K. Peng, M. R. Jackson, J. A. Rongong, F. L. Chu, and R. M. Parkin, "On the energy leakage of discrete wavelet transform," Mechanical Systems and Signal Processing, vol. 23, no. 2, pp. 330343, 2009. 

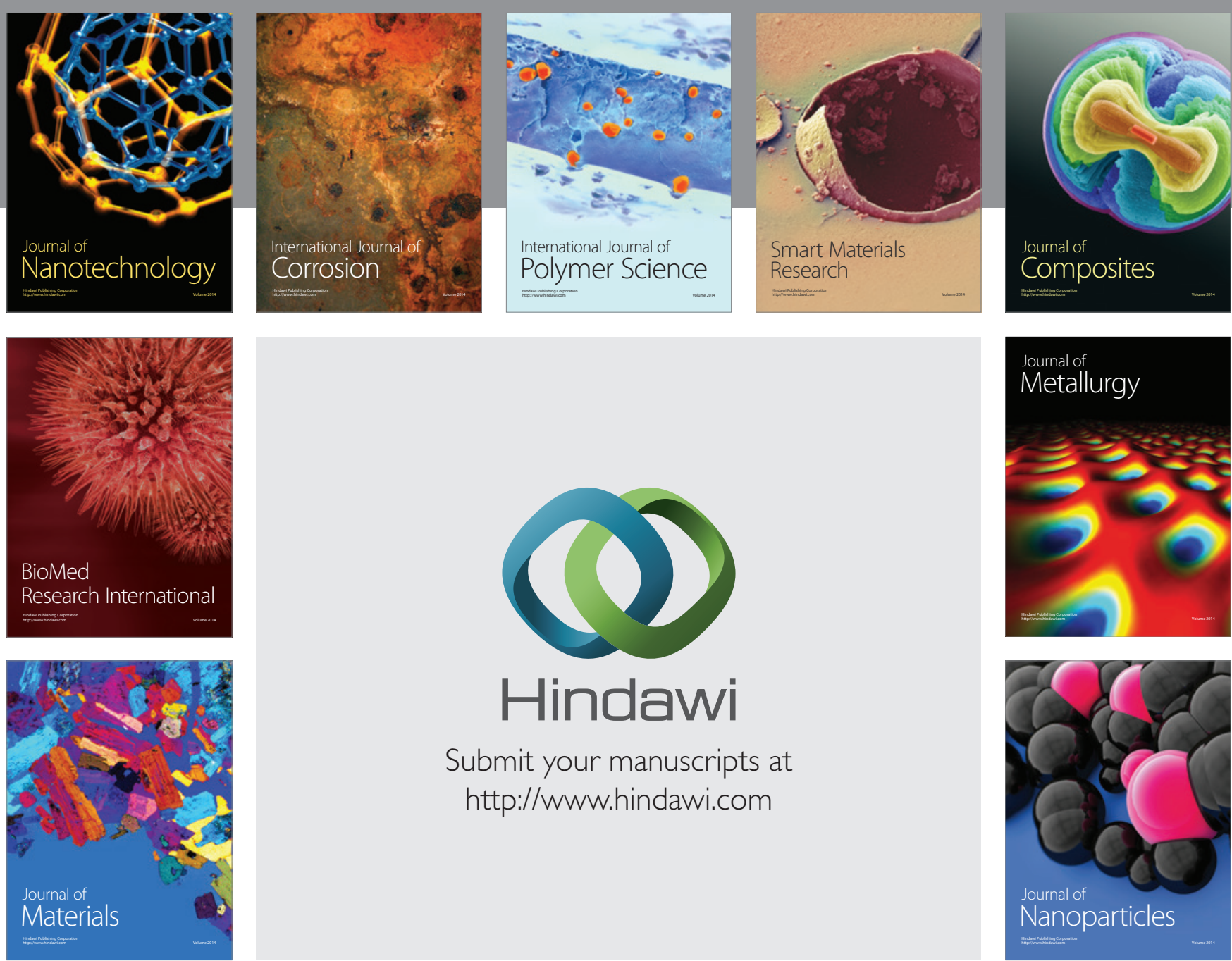

\section{Hindawi}

Submit your manuscripts at

http://www.hindawi.com

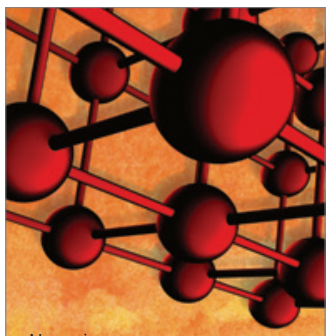

Materials Science and Engineering
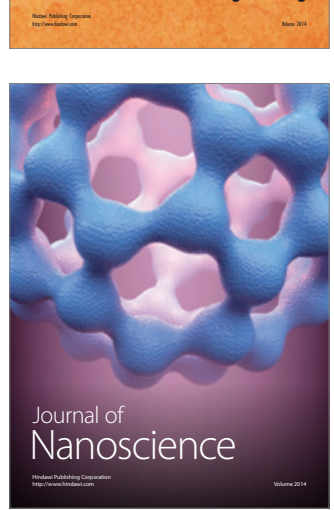
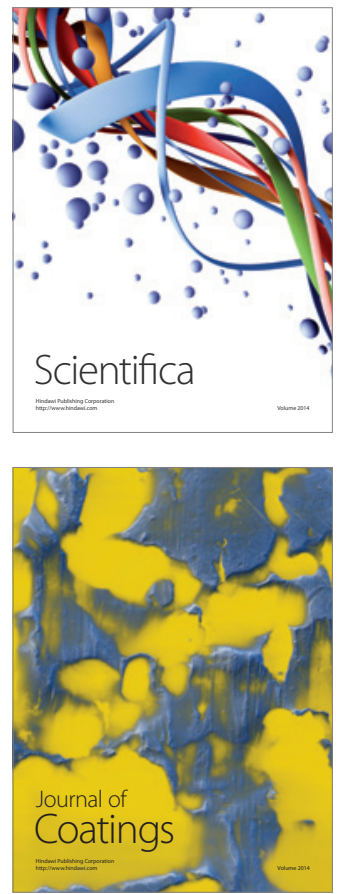
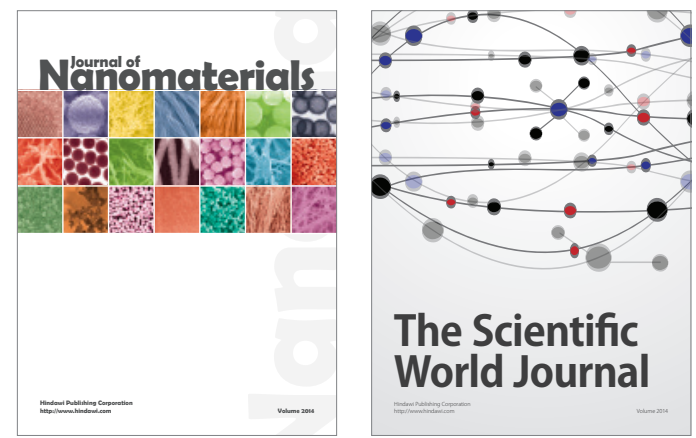

The Scientific World Journal
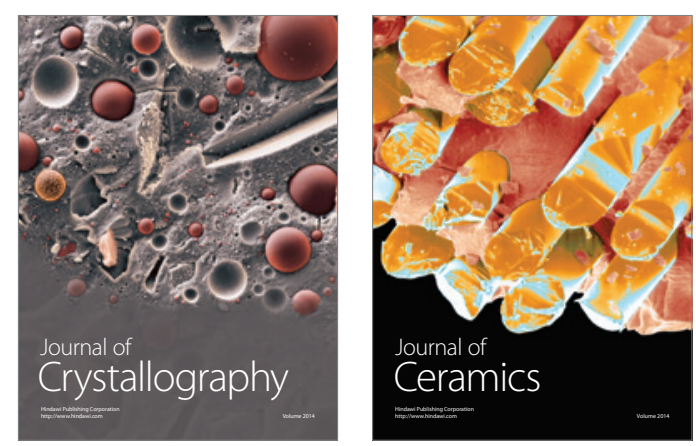
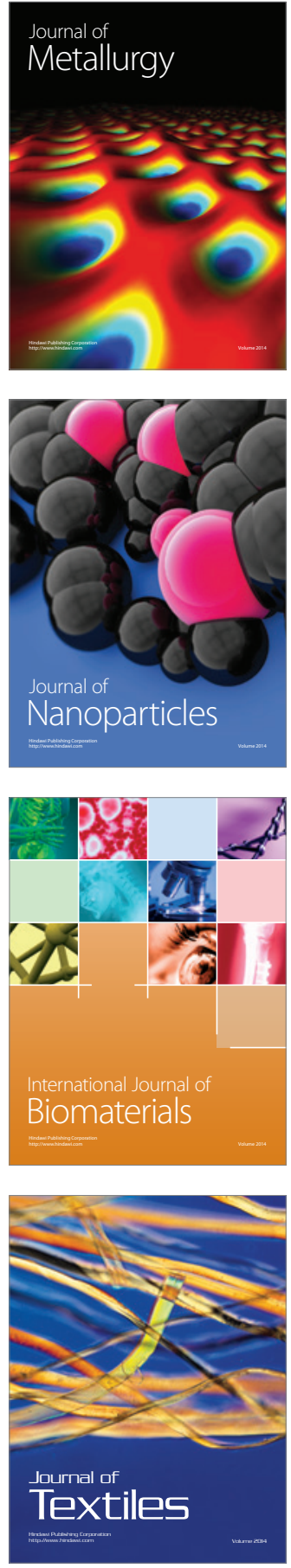